\section{A new grading system for assessing orbicularis muscle function}

R Rahman, A Berry-Brincat and VT Thaller

different levels of experience can use it reliably.

Eye (2003) 17, 610-612. doi:10.1038/

sj.eye. 6700444

Keywords: orbicularis muscle; essential blepharospasm; grading system; botulinum toxin

\section{Introduction}

Benign essential blepharospasm and hemifacial spasm are forms of facial dyskinesia. Both conditions produce debilitating episodic contractions of the facial musculature notably in the orbicularis oculi. The main objective in the treatment of blepharospasm and hemifacial spasm is to abolish or decrease unwanted, repeated forced closure of the eyelids.

Currently, this is best achieved by the use of botulinum toxin injections to the orbicularis oculi muscles. When botulinum toxin is administered for the first time at fixed doses, variable clinical responses can be observed in patients with the same form of dystonic disorder. ${ }^{1}$ Many factors may contribute to this phenomenon, including variability in the rate of absorption of the drug. ${ }^{1}$ Marked inter- and intrapatient variability has been found in the length of effect of botulinum toxin. ${ }^{2}$ Long-term changes in the duration of relief with botulinum toxin treatment of essential blepharospasm and hemifacial spasm have been reported. ${ }^{2}$

It would be most useful to clinicians to be able to quantify the effectiveness of botulinum toxin treatment. Fahn ${ }^{3}$ first proposed two rating scales for blepharospasm. The blepharospasm rating scale serves to quantitate the severity of the blepharospasm and to indicate qualitatively the muscles involved with involuntary contractions. The blepharospasm disability scale (BDS) quantitates the severity based on functional impairment caused by blepharospasm by evaluating the impact of 
blepharospasm on the activities of daily living. In most studies, ${ }^{2,4}$ effectiveness of treatment with botulinum toxin injections is assessed by 'relief 'or 'improvement' of symptoms without specifying the nature of the improvement. Currently, toxin treatment is planned entirely on patients' reported symptoms. These are often variable, and may be unreliable, and are influenced by mood and circumstances.

\section{Methods}

The following scale has been used by one of the authors (VTT) in his botulinum toxin clinic for several years to grade orbicularis function.

Grade 0: Incomplete eyelid closure.

Grade 1: Lids just closing, minimal resistance to overcome.

Grade 2: Closing well, some resistance, can be easily overcome.

Grade 3: Strong closure, can be overcome with difficulty.

Grade 4: Very strong closure, cannot be overcome or overcome with extreme difficulty.

Grade 0 is assessed wholly by visual observation; grade 1 assessment is partially visual and partially tactile; and grades $2-4$ assessment is wholly tactile.

\section{Testing procedure}

1. To assess grades $1-4$, the subjects are asked to close their eyes gently. The observer's index finger is placed on the subject's upper lid and the thumb on the lower lid, pretarsally. The observer may need to use the other fingers to gently lift any upper lid skin fold that interferes with pretarsal finger placement.

2. The subject is then instructed to squeeze his/her eyes as tightly shut as possible, resisting maximally the observer's attempts to open them.

3. While the subject is squeezing, the observer attempts to open the eyes using the preplaced fingers, noting how much effort is required.

Ethical approval was obtained for the study and toxin clinic patients were invited to participate. They were only included in the study after informed consent was obtained. In order to establish the interobserver agreement, one consultant ophthalmologist (VTT), three ophthalmologists in training, and one nurse practitioner evaluated the same 65 patients, undergoing treatment with botulinum toxin for essential blepharospasm and hemifacial spasm. Observers assessed orbicularis function by asking each patient to close both eyes forcefully, while the observer tried to open them manually. The measure of agreement across the observers was estimated by $\kappa$ statistics. The Kruskal-Wallis nonparametric test was used to assess the difference between the observers and a $P$-value of $<0.05$ would indicate differences between the observers.

\section{Results}

A total of 130 eyes of 65 patients were assessed. Of these, $52(80 \%)$ patients were female.The age range was 37-88 years (average age 65 years). Overall, the interobserver agreement $(\kappa=0.52$ ) was satisfactory. Comparing interobserver concordance for the right and left eyes separately ( $\kappa=0.54$ for right; $\kappa=0.52$ for left) showed the same degree of agreement.

The Kruskal-Wallis nonparametric test was used to assess the difference between the observers and a $P$-value of $<0.05$ would indicate difference between the observers. However, in this case we cannot reject the null hypothesis (of equal observer medians) as we have a $P$-value of 0.84 for the right and 0.49 for the left eyes. Since both $P$-values were well above 0.05 , this indicated good consistency between observers when using this rating system.

\section{Discussion}

We have analysed the interobserver agreement of the orbicularis grading system and found good correlation among all observers. This grading system determines treatment outcomes of botulinum toxin injections primarily by measuring an objective feature, that is, weakness of the orbicularis muscle. Although it is more informative and less dependent on patients's self-reports, it still relies on patient cooperation in squeezing their eyes.

Objective assessment of botulinum toxin effectiveness is important in planning future treatment dosages as patients easily confuse disease symptoms and toxin treatment side effects. The senior author tests and records the orbicularis function of all patients receiving eyelid botulinum toxin prior to every treatment. The orbicularis score confirms whether and to what degree the toxin has been effective in weakening the orbicularis. Patients sometimes assert that the toxin injections are no longer working, or that the last batch was no good, and it is important to determine objectively whether this is indeed the case. Usually, one is able to reassure the patient that although symptom control may not have been adequate, the toxin is still working. It is also helpful in identifying the few patients who develop immunity to botulinum toxin A. 
While the scale was primarily developed for use in the toxin clinic, it is also useful in ocular myopathy in assessing the likelihood of post ptosis surgery corneal exposure.

Our study shows that the orbicularis function assessment is reasonably reproducible and relatively easy to quantify by a physician. It has been found useful in the day-to-day management of patients with facial dyskinesia.

\section{Conclusions}

A simple, new, five-point, clinical grading system for orbicularis muscle function is presented. Medical staff with different levels of experience can use it reliably. The scoring relies on combined visual inspection and tactile muscle strength assessment. Orbicularis function has been found useful in planning and assessing botulinum toxin treatment of essential blepharospasm and hemifacial spasm.

\section{References}

1 Eleopra R, Tugnoli V, De Grandis D. The variability in the clinical effects induced by botulinum toxin type A: the role of muscle activity in humans. Mov Disord 1997; 12(1): 89-94.

2 Ainsworth JR, Kraft SP. Long-term changes in duration of relief with botulinum toxin treatment of essential blepharospasm and hemifacial spasm. Ophthalmology 1995; 102: 2036-2040.

3 Fahn S. Rating scales for blepharospasm. Adv Ophthal Plastic Reconstruct Surg 1985; 4: 97-101.

4 Taylor JDN, Kraft SP, Kazdan MS et al. Treatment of blepharospasm and hemifacial spasm with botulinum A toxin: a Canadian mulitcentre study. Can J Ophthalmol 1991; 26(3): 133-137. 